\title{
Analysis of CNC engraving process of New Chinese style furniture parts
}

\author{
Junhua $\mathrm{Wu}^{1, \text { a }}$, Weihong Sun ${ }^{2, \mathrm{a}}$, Honggang Zhao*1, a \\ ${ }^{1}$ Wood Material Science and Engineering Key Laboratory of Jilin Province, Beihua University, Jilin \\ 132013, China
}

${ }^{2}$ CNPC Northeast Refining \& Petrochemical Engineering Co.Ltd JiLin Design Institute, Jilin 132002, China

a54086442@qq.com

*corresponding author

Keywords: New Chinese style furniture; CNC engraving; pattern design; digital modeling

Abstract. New Chinese style furniture is the cultural significance of Chinese traditional furniture in a contemporary context interpretation. It is the use of modern technologies, equipment, materials, and processes for production and processing, which can meet standardization and generalization of modern furniture requirements. Engraving is an important process for furniture parts of Chinese furniture. With the rapid development of computer and automatic control technology, traditional hand-carving has been gradually replaced by $\mathrm{CNC}$ technology. Furthermore, CNC engraving plays an incomparable advantage in many aspects, including pattern design, engraving quality and process efficiency, etc. And it is the future trends in furniture design and manufacturing.

\section{Introduction}

Modern Chinese style furniture is to use modern technology, equipment, materials and technology for production and processing, which is in line with the standard and general requirements of modern furniture, embodies the time spirit; meanwhile, with the rich national characteristics and traditional culture connotation, to adapt to mass production of industrialization[1]. Modern Chinese style furniture is the cultural significance of Chinese traditional furniture which is developed in the current era, so it is also called New Chinese style furniture[2].

Furniture parts carving processing is an important process of Chinese style furniture, the traditional manual engraving due to production efficiency is low, can not repeat the production, and the level of the carving master is higher, it is difficult to meet the requirements of mass production, more and more control of industrial production, and therefore the numerical control engraving technology has become the development trend of Chinese furniture surface decoration technology.

With the rapid development of computer technology and automatic control technology, laser engraving technology, numerical control engraving technology and other advanced technology means in the furniture design and manufacturing industry has been widely used, greatly improving the efficiency of furniture products processing. However, with the computer aided design and manufacturing and $\mathrm{CNC}$ machining technology for the characteristics of $\mathrm{CNC}$ engraving process have become increasingly evident.

\section{A brief introduction to numerical control engraving software}

At present, there are many CAD/CAM computer aided design and manufacturing software, such as PRO/E, Art, cam UG, JDpaint and so on. A comprehensive comparison of the CAD / CAM 
software, considering our country the furniture enterprise production status and pattern carving, this paper carved jdpaint software as an example, application of carving analysis, focus on the carving design, engraving quality and processing efficiency of factors were discussed in detail.

JDpaint software is the development of Beijing carved company, it is the basic part of CNC engraving system.It is not only a powerful engraving software, also an open CAD / CAM software product development platform. The virtual sculpture software (JDVirs) is based on the platform for the development of professional software.It provides a possiblity for digital design of the carving patterns, which simulates manual carving process[3,4]. Started by the two-dimensional line release in the computer, by coloring, stamping, to the material, polishing and other methods in a flat, completing wood carving in the bottom off, making bricks, refinement, resulting in computer design exquisite wood carving model, and the design of the model is machined by CNC engraving machine. At present, this small tool fine engraving machine is suitable for wood engraving. Futhermore, it has obvious advantages for the complex Chinese furniture carving pattern and small carved trim batch processing[5].

\section{The CNC carving process of furniture pattern design}

CNC carving process is made of four steps, including furniture patterns design, digital modeling, tool path generation and processing. Fig. 1 is a flow chart of CNC engraving of furniture parts. Following the case of furniture carved door panels, combined with the furniture factory producing actual, the detail of CNC machining process is analyzed. Engraving pattern's name is Xishangmeishao, engraving size: $280 * 750 \mathrm{~mm}$, engraving depth of $5 \mathrm{~mm}$.

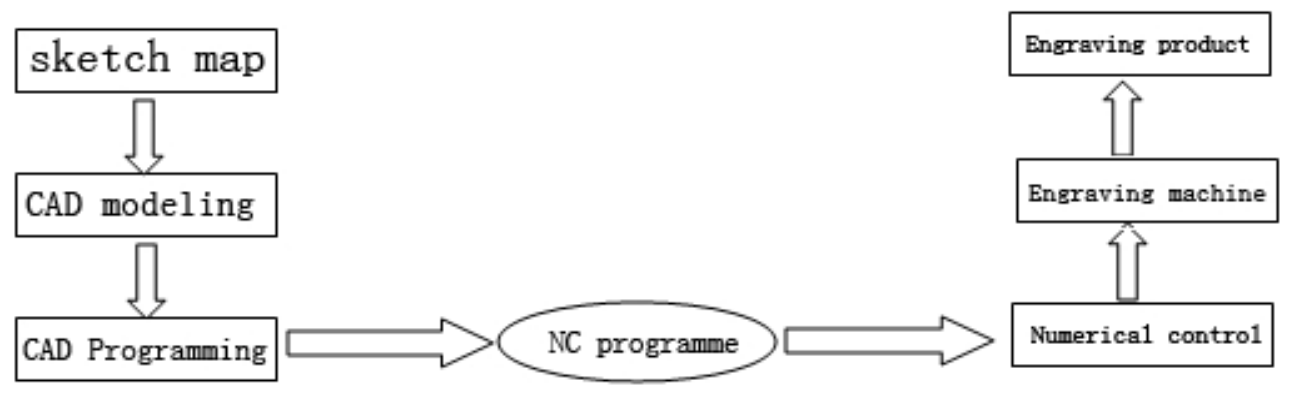

Fig.1. Flow chart of CNC engraving

(1) Pattern design

Designs are two-dimensional pattern design that just using computer software to implement a design drawing, and it is similar to hand-carved designs. You can also put in practice by hand-painted designs on paper firstly, then enter it into the computer for processing, and use Jdpaint software to draw it finally. Design is simple and easy, and can be modified time and again.

(2) Digital modeling

Digital modeling is good for designing two-dimensional graphics for coloring, stamping, cutting, polishing and other virtual processing, equivalent to simulate the whole process of hand-carved in the software and computer virtual hand-carved into sculptures. Digital modeling of design pattern is shown in Figure 2. Engraving shapes and depth can be appeared in different colors. Different color shades represent different engraving depth of carvings pattern. Designers can modify the detail place repeatedly if not satisfied with it through a global observation of the graphic. 


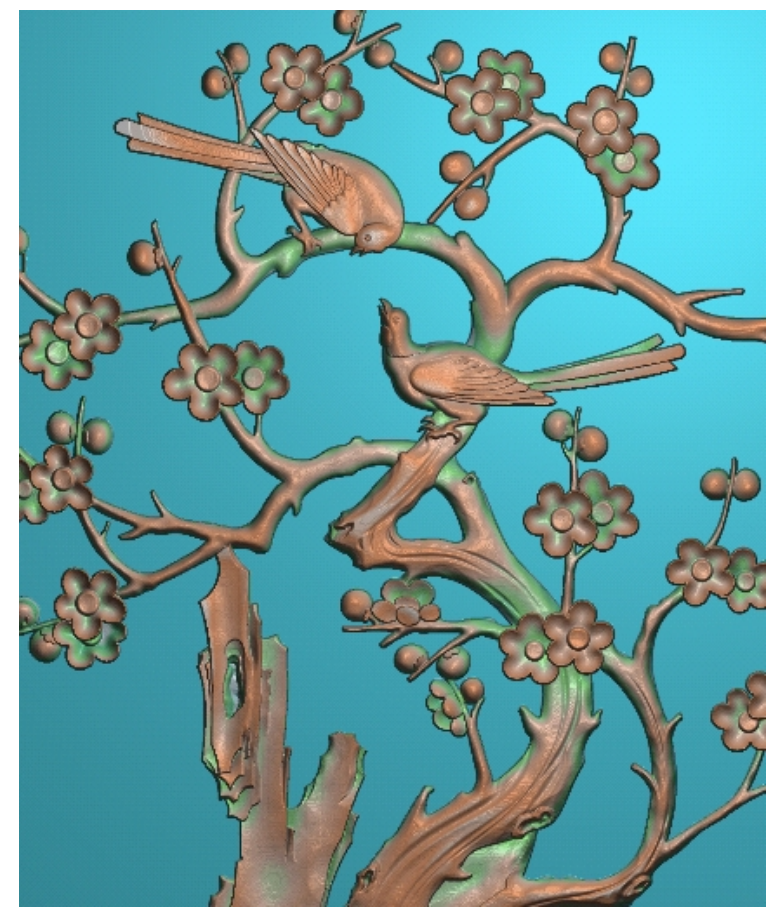

Fig.2. Digital modeling of design pattern

(3) Tool path generation

Generating the tool path is equivalent to the NC programming implementation process of a carving pattern which is digital modeled previously. Jdpaint has a special path Wizard panel, and the user just needs make a choice of a certain amount of processing technology by generating the tool path. Its operation is simple, easy to use, and the requirements of numerical control programming is been greatly reduced for designers. Table 1 lists tool path parameters of surface engraving process. Just through a simple training, users are able to achieve a higher level of path generation. It can generate very high machining efficiency paths through appropriate choice of parameters of interfaces. In this example, processing paths include the embossed surface path and cutting path of the workpiece contours.

Tab.1. Tool path parameters of surface processing

\begin{tabular}{llcc}
\hline processing size & tool path mode & tool model & $\begin{array}{c}\text { engraving } \\
\text { depth }\end{array}$ \\
\hline $280 * 750 * 5 \mathrm{~mm}$ & intricate engraving & JD-30-0.2 & $5 \mathrm{~mm}$ \\
\hline
\end{tabular}

(4) Process of implementation

Process of implementation is output into the resulting tool path of NC machine file format which is simulated by NC machining system software and can be completed by a CNC engraving machine. Machines for carved parts furniture is mainly for Saidiao series CNC carving machine. Machining spindle speed and feed rate can be set in the NC software to control the engraving speed and quality of the carving product.

\section{Characteristics analysis of $\mathrm{CNC}$ engraving}

\section{Pattern design}

Furniture pattern engraving pattern design is a process of digital modeling, which is a pattern 
design of three dimensional digital modeling based on a two-dimensional plane[6]. It has the following advantages compared with the traditional hand-carving.

(1) Pattern design has high efficiency, and easy to modify and can be reused. Computer mouse design can save work and time, and greatly improving efficiency, instead of manual operations. As engraving model works of Xishangmeishao, skilled drawing staff generally can complete it in less than two hours of virtual model of the engraving. It has more incomparable advantages in making geometric patterns, and pattern of any complex shape can be processed. We can observe its effects directly on the computer, and easy to modify. And you can avoid human errors. Engraving pattern design is more and more diverse; this can give full play to the ingenuity of the designer.

(2) Easily build rich Engraving model libraries, so that when designing a new product you can feel free to call. Digital model can be reused multiple times. At the time of the development of new products, we can bring up similar models from the model library and modify it. We can also complete multiple patterns design which can be redeployed from the model base. So it owns strong advantages both in terms of quantity of model fodder and design flexibility.

(3) Virtual sculpture has many efficient professional features, such as the Dragon scale common in antique furniture surface, lucky clouds, v-cutter line of grain patterns, which is simulated a generic hand-engraving techniques in substance. In effect, it has easy and fast modeling features. This can meet the requirements of manual carving skills. The Software can be developed more professional in pattern modeling capabilities according to customer's requirements.

\section{Pattern carving quality}

Traditional hand-carved quality generally depends on the master's skill level, and a master with a better level can carve out a lively and charming design pattern. We must be strict in quality of each process. Moreover, Polishing craft is very important for engraving patterns which sensitivity and charm affected by its quality. Because of good quality, more stable, precise, and short processing time, CNC engraving become more and more popular. In particular, good surface finish products only require a short time for polishing in the later. For batch processing, it has the consistency of the different products. Furthermore, with regard to the big embossment works, higher consistency of segmented convergence before and after engraving that can be revealed.

\section{Conclusions}

With the rush of people's traditional culture, new Chinese furniture plays an increasingly higher proportion in the furniture market. Engraving is an important new Chinese furniture decoration; it made a rapid progress with the development of science and technology. It helps furniture enterprises to improve production efficiency and reduce production costs at the same time. Although many furniture companies are common in a pattern which mainly in hand-carved, mechanical processing as a supplement, CNC engraving process has incomparable advantages including in achieving industrialization and mass production of furniture parts and decorative patterns. Moreover, it is the future trend in the furniture industry.

\section{Acknowledgements}

This work was financially supported by the Postdoctoral research projects starting Fund of Jilin province, scientific flat roof research project of Jilin province, and the key scientific and technological project (20150204038NY) of science and technology department of Jilin Province. 


\section{References}

[1] Lijuan Shen. Discussion on the mode of modern Chinese-style house and traditional culture [J], Public science and technology, 2006.1.

[2] Wenjuan Xie. Analysis of modern Chinese style furniture design ideas[D], Huazhong University of science and technology, 2008.5.

[3] Jingjing Jiao, Ruo Zhang. Analysis on the carved modern technology of furniture pattern[J], Light industry technology , 2012(8):100-101.

[4] Huilan Wu, Weimin Zen. Computer numerical control carving technology and its application prospect[M], Mechanical design and manufacturing, 2007(1):159-160.

[5] Nianxiang Wang. Sculpture art of Ming-style furniture [M] Beijing: Beijing Arts and crafts press, 2001.1.

[6] Lean Cai.Art carved surface modeling and carving. Metal processing, 2005(5):72-73. 